\title{
Valley trion dynamics in monolayer $\mathrm{MoSe}_{2}$
}

\author{
Feng Gao, ${ }^{1}$ Yongji Gong, ${ }^{2,3}$ Michael Titze, ${ }^{1}$ Raybel Almeida, ${ }^{1}$ Pulickel M. Ajayan, ${ }^{2,3}$ and Hebin Li ${ }^{1, *}$ \\ ${ }^{1}$ Department of Physics, Florida International University, Miami, Florida 33199, USA \\ ${ }^{2}$ Department of Chemistry, Rice University, Houston, Texas 77005, USA \\ ${ }^{3}$ Department of Materials Science and NanoEngineering, Rice University, Houston, Texas 77005, USA
}

(Received 11 May 2016; revised manuscript received 16 October 2016; published 12 December 2016)

\begin{abstract}
Charged excitons called trions play an important role in the fundamental valley dynamics in newly emerging two-dimensional semiconductor materials. We use ultrafast pump-probe spectroscopy to study the valley trion dynamics in a $\mathrm{MoSe}_{2}$ monolayer grown by using chemical vapor deposition. The dynamics displays an ultrafast trion formation followed by a nonexponential decay. The measurements at different pump fluences show that the trion decay dynamics becomes slower as the excitation density increases. The observed trion dynamics and the associated density dependence are a result of the trapping by two defect states as being the dominating decay mechanism. The simulation based on a set of rate equations reproduces the experimental data for different pump fluences. Our results reveal the important trion dynamics and identify the trapping by defect states as the primary trion decay mechanism in monolayer $\mathrm{MoSe}_{2}$ under the excitation densities used in our experiment.
\end{abstract}

DOI: 10.1103/PhysRevB.94.245413

\section{INTRODUCTION}

Layered transition metal dichalcogenides (TMDs), $M X_{2}$ $(M=\mathrm{Mo}, \mathrm{W} ; X=\mathrm{S}, \mathrm{Se}, \mathrm{Te})$, represent a new class of atomically thin two-dimensional (2D) materials inspired by the discovery of graphene [1]. In contrast to graphene, monolayer TMD has a direct band gap [2,3] in the visible region and displays strong photoluminescence (PL) at the $K$ and $-K$ points in the Brillouin zone [4,5]. Moreover, the inversion symmetry breaking and strong spin-orbit coupling in monolayer TMD lead to contrasting circular dichroism in the $\pm K$ valleys [6]. Consequently, the interband transitions at the two valleys can be selectively excited by an optical field with proper helicity. This ability to selectively address different valleys enables optical generation, control, and detection of valley polarizations [7-10] and valley coherence [11]. These unique properties and recent advances in sample fabrication [12] make layered TMDs promising materials for novel applications in optoelectronics [13] and the field of valleytronics [14-20].

The optical excitation of semiconductors creates excitons which are electron-hole pairs bound through Coulomb interactions. In the presence of excess charges, charged excitons called trions [21,22] can be formed through the coalescence of an exciton and a free charge or directly from an unbound electron-hole plasma [23]. In monolayer TMDs, strong Coulomb interactions lead to exceptionally high binding energies for excitons [24] and trions [11,25,26], allowing them to exist even at room temperature. As excitons, trions also have significant influences on the optical and electronic properties of layered TMDs. For example, trions can modify the overall PL spectrum [26] in monolayer $\mathrm{MoSe}_{2}$ and reduce the conductivity [27] in monolayer $\mathrm{MoS}_{2}$. The interplay between exciton and trion is critical. The trion state has a lower energy and provides a relaxation channel for excitons. Trions can be excited by an optical phonon into an excitonic state to realize a upconversion process [28] in monolayer $\mathrm{WSe}_{2}$. Coherent exciton-trion coupling has also been observed [29] in monolayer $\mathrm{MoSe}_{2}$. Moreover, trions play an important role in applications such as

\footnotetext{
*hebin.li@fiu.edu
}

quantum information processing [30]. Therefore, a thorough understanding of the fundamental carrier dynamics in monolayer TMD has to include both exciton and trion dynamics.

The experimental investigation of the carrier dynamics upon an optical excitation has been the focus of recent studies based on techniques such as time-resolved PL [31,32], ultrafast pump-probe spectroscopy [33-43], optical Kerr spectroscopy [44,45], coherent 2D spectroscopy [46], and hole-burning spectroscopy [47]. These studies have revealed valuable structure and dynamic information in layered TMD. However, the focus has been mainly on the exciton dynamics while the trion dynamics remains largely unexplored. Recent studies of the trion formation in monolayer $\mathrm{MoSe}_{2}$ by pumpprobe spectroscopy [43] and the trion emission in monolayer $\mathrm{WSe}_{2}$ by time-resolved PL [48] have brought attention to the valley trion dynamics in layered TMDs.

Here we report a study of the valley trion dynamics in a chemical-vapor-deposition (CVD) grown $\mathrm{MoSe}_{2}$ monolayer by using ultrafast pump-probe spectroscopy. We find that the trion population decays nonexponentially after an ultrafast $(<500 \mathrm{fs})$ trion formation from photoexcited free carriers. The trion decay can be fit with a biexponential decay function. The measurements at different pump fluences show a surprising density dependence of the trion decay: The dynamics becomes slower as the excitation density increases. We present a theoretical model based on a set of rate equations that reproduces the experimental data quantitatively for all pump fluences. The model shows that the primary mechanism responsible for the observed dynamics and density dependence is the fast and slow trapping by two defect states. The slower dynamics at higher densities is due to the limited density of the defects. The result supports the trion formation explanation [27] of the reduced terahertz conductivity and its corresponding dynamics in monolayer $\mathrm{MoS}_{2}$.

\section{SAMPLE AND EXPERIMENTAL METHOD}

Monolayer $\mathrm{MoSe}_{2}$ was synthesized by using the CVD process as described in detail in a previous report [12]. Monolayer flakes were grown on a $\mathrm{SiO}_{2} / \mathrm{Si}$ substrate and 

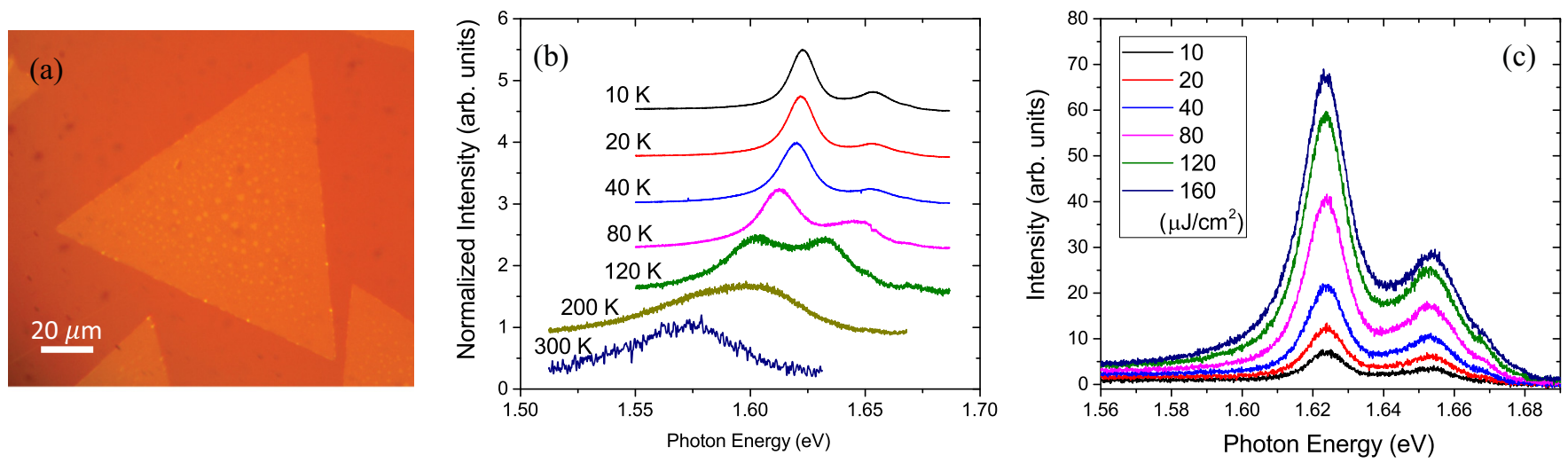

FIG. 1. (a) The optical image of a CVD MoSe 2 monolayer. (b) Normalized PL spectra at various temperatures from 10 to $300 \mathrm{~K}$. The spectra are shifted vertically for clarity. (c) PL spectra at $10 \mathrm{~K}$ with various pump fluences from 10 to $160 \mu \mathrm{J} / \mathrm{cm}^{2}$.

transferred to a glass substrate. The optical image of a typical monolayer $\mathrm{MoSe}_{2}$ flake is shown in Fig. 1(a). The flakes are equilateral triangles with sides ranging from tens of $\mu \mathrm{m}$ to more than $100 \mu \mathrm{m}$. The sample was placed in a microscopy liquid helium cryostat (Cryo Industries of America RC102CFM) for low temperature experiments. The sample was first characterized with PL measurements at different temperatures. Femtosecond pulses with a central wavelength at $720 \mathrm{~nm}$ were used as the pump for PL experiments. The PL spectra at various temperatures from 10 to $300 \mathrm{~K}$ are shown in Fig. 1(b). As the temperature decreases, the PL spectrum changes from a broad single peak to two separate peaks with an energy difference of $\sim 30 \mathrm{meV}$. At low temperatures, the peak at the higher energy $(1653 \mathrm{meV}$ at $10 \mathrm{~K})$ is identified as the $A$ exciton resonance while the one at the lower energy (1623 meV at $10 \mathrm{~K}$ ) as the trion resonance. This assignment is consistent with previously published studies $[26,29,43]$ of the PL spectra, the resonance energies, and the trion binding energy. The PL was also measured under different pump fluences to check how the excitation density affects the exciton and trion resonances. Figure 1(c) shows the PL spectra at $10 \mathrm{~K}$ under various pump fluences. The spectra are fit to a Lorentzian double peak profile to extract peak positions, linewidths, and peak areas (see the Supplemental Material [49] for details). As the pump fluence changes from 10 to $160 \mu \mathrm{J} / \mathrm{cm}^{2}$, the exciton and trion resonance energies have a small variation of 1.5 and $0.4 \mathrm{meV}$, respectively. The linewidth increases from 15.6 to $18.1 \mathrm{meV}$ for the exciton and from 13.1 to $16.7 \mathrm{meV}$ for the trion. While both exciton and trion intensities increase linearly with the pump fluence, the ratio of exciton to trion peak area decreases from 0.52 to 0.38 . This ratio can be used to determine the distribution of the initial carrier density between the exciton and trion states.

The ultrafast pump-probe experiment was performed with the setup shown in Fig. 2(a). A Ti:sapphire femtosecond oscillator (Coherent Mira 900) and an optical parametric oscillator (OPO) (Coherent Mira OPO) were used to provide the probe and pump pulses, respectively. The pulse duration is $\sim 500 \mathrm{fs}(\sim 200 \mathrm{fs})$ for the pump (probe) and the repetition rate is $76 \mathrm{MHz}$. The pump pulse is tuned to $1722 \mathrm{meV}$ to excite free carriers slightly above the $A$ exciton energy but below the $B$ exciton energy. The probe pulse is tuned to probe at the peak of the trion resonance at $1623 \mathrm{meV}$. The bandwidth of the probe pulse $(\sim 9 \mathrm{meV})$ is much larger than the variation in the trion resonance energy under different pump fluences at a temperature of $10 \mathrm{~K}$. The pump and probe beams are focused on the sample by a long working distance $50 \times, 0.55$ numerical aperture (NA) objective lens (Mitutoyo Plan APO 50× ). On the sample, the pump has a spot size of $7 \mu \mathrm{m}$ in diameter and the probe spot is $5 \mu \mathrm{m}$ in diameter. The probe fluence is $1 \mu \mathrm{J} / \mathrm{cm}^{2}$ and the pump fluence is varied from 10 to $160 \mu \mathrm{J} / \mathrm{cm}^{2}$. We tested the probe fluence of $2 \mu \mathrm{J} / \mathrm{cm}^{2}$ and found no noticeable difference in the measured dynamics. The pump and probe beams are circularly polarized to selectively excite and probe the transition at one of the $\pm K$ valleys depending on the helicity. The reflected probe beam is collected by the same objective lens and recorded with a photodetector and a lock-in amplifier while the pump beam is modulated by a chopper. The time delay of the probe pulse can be varied by a delay stage. A pump-probe spectrum is acquired by measuring the reflected probe as a function of the time delay between the pump and probe pulses. The PL measurements were also done in the same setup with the probe beam being blocked. The PL signal was collected by a half-meter spectrometer (Horiba iHR550) and recorded on a CCD camera (Horiba SYN-2048X512) cooled by a thermoelectric cooler.

\section{RESULTS AND DISCUSSIONS}

Representative pump-probe spectra are shown in Fig. 2(b) for a sample temperature of $10 \mathrm{~K}$ and a pump fluence of $80 \mu \mathrm{J} / \mathrm{cm}^{2}$. The blue (red) circles are the data obtained with the cocircularly (cross-circularly) polarized pump and probe beams, that is, the pump and probe polarizations are $\sigma^{+} \sigma^{+}$ $\left(\sigma^{+} \sigma^{-}\right)$. The pump-probe spectra feature a fast rising followed by a decay of the signal up to hundreds of ps.

To investigate the rising signal, Fig. 2(c) shows a segment of several ps around the zero time delay. In addition to the pump-probe spectra, a 500-fs Gaussian pulse is plotted as a dashed line. The normalized time integral of the Gaussian pulse, shown as a solid line, matches well with the rising slope of the pump-probe spectra. Therefore, the rising time of the signal is limited only by the time resolution of the pump pulse. For the cocircular configuration, this indicates that the trion formation time is within $500 \mathrm{fs}$. This time is consistent with the trion formation time and its dependence on 
(a)

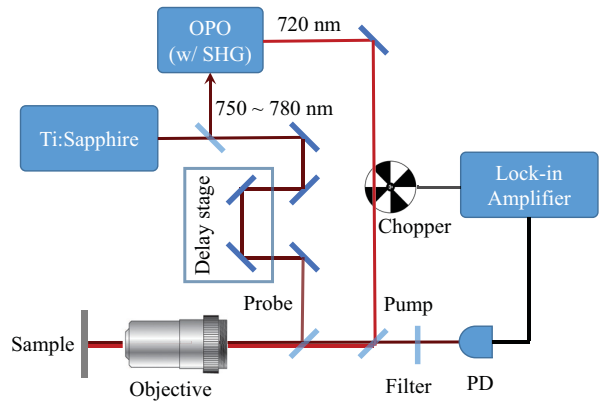

(b)

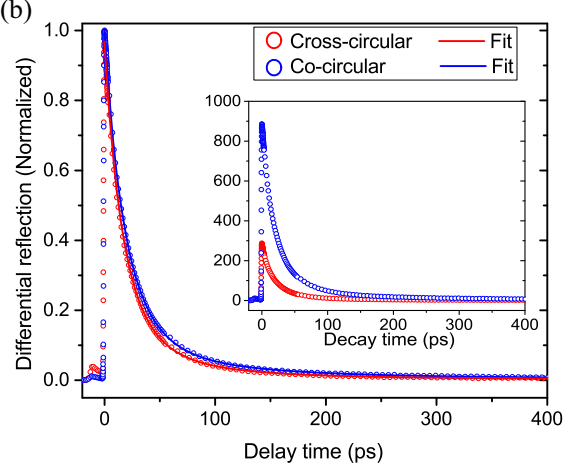

(c)

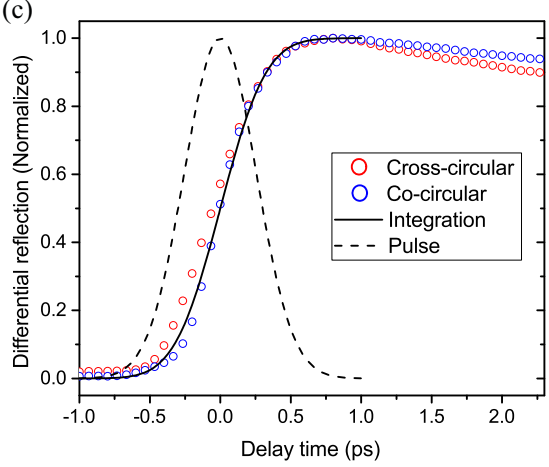

FIG. 2. (a) The schematic of the pump-probe experiment. (b) Normalized pump-probe spectra with cross-circular (red circles) and cocircular (blue circles) polarizations at $10 \mathrm{~K}$ with a pump fluence of $80 \mu \mathrm{J} / \mathrm{cm}^{2}$. The solid lines are fit to a biexponential decay function. The inset shows the spectra without normalization. (c) A zoom-in showing the rising slope. The dashed line is a Gaussian pulse with a duration [full width at half maximum (FWHM)] of $500 \mathrm{fs}$. The solid line is the normalized integration of the Gaussian pulse.

the excitation energy reported by Singh and coauthors [43]. The variation in the trion formation time can be caused by the differences in doping density, excitation energy, and excitation power.

The normalized spectrum with the cross-circular configuration shows a nearly identical rising signal. This can be a result of two possible mechanisms: (1) No or negligible valley polarization is created initially by the pump pulse; and (2) a finite polarization is generated but decays rapidly due to ultrafast intervalley relaxation. The valley polarization can be studied with helicity-resolved PL spectra. Observations of a persistent polarization in the PL spectra have been reported for excitons in $\mathrm{MoS}_{2}$ [8-10,32], $\mathrm{WSe}_{2}$ [11,41], and $\mathrm{WS}_{2}$ [50], and trions in $\mathrm{WSe}_{2}$ [48]. The reported circular polarization degree is considerably less than $100 \%$ in most experiments. The imperfect but persistent polarization can be explained with an ultrafast initial polarization decay due to fast intervalley relaxation, in which some carriers lose the initial polarization quickly while others maintain for a longer time. However, our time-integrated PL measurement and a previous PL study [51] show nearly zero valley polarization in monolayer $\mathrm{MoSe}_{2}$, unlike other TMDs. Since the time-integrated PL measurement has no time resolution and the time-resolved PL spectrum [51] obtained with a streak camera has a time resolution of 4 ps, the PL measurement does not provide information about the polarization dynamics within $4 \mathrm{ps}$, therefore insufficient to determine the exact mechanism [51]. In our experiment, the amplitude of the pump-probe spectrum, prior to the normalization, with the cross-circular polarizations is about $30 \%$ of the amplitude with the cocircular polarizations, as shown in the inset of Fig. 2(b). This observation indicates a finite initial polarization is created. The reduced trion intensity for the cross-circular polarizations has also been observed in the PL [48] spectra. The time-resolved PL measurements [32,48] suggested an intervalley relaxation time shorter than the time resolution of $4 \mathrm{ps}$ in their experiments. Our data show that the intervalley relaxation time is shorter than 500 fs and only a part of the carrier population is involved in this ultrafast initial intervalley relaxation.

It has been shown [23] that the trion formation has two possible channels. In the presence of excess free charges, an exciton can capture an extra charge to form a trion. This is the primary process for trion formation at low carrier densities. At a sufficiently high density, trions can be formed directly from unbound carriers through a three-particle formation process. In our experiment, the trion formation is as fast as the exciton formation within the time resolution of our measurement. We suspect that the trion formation at the short time scale is mainly the three-particle formation from free carriers. However, our results cannot rule out the possibility of an ultrafast ( $<500 \mathrm{fs}$ ) exciton-to-trion formation process. As we will show in the simulation, a slow trion formation process through the coalescence of excitons and excess charges may exist and lead to a better agreement between the simulated and experimental data at long delay times.

After the rise, the pump-probe signal displays a nonexponential decay which includes a fast decay at the time scale within tens of ps and a slower decay at hundreds of ps. Similar behaviors in the decay signal have been observed for both excitons [31-33,35,36,38,41,42] and trions [48] in various TMD monolayers. A biexponential decay function provides a good fit to the data in some cases [31,32,35,36,41] while other experiments $[33,38,42]$ suggest that a triexponential decay function is required. In our experiments, a biexponential decay function is sufficient to fit the decay signal. To analyze the decay dynamics, we use the biexponential decay function, $y=A_{0}\left(e^{-t / t_{1}}+A_{21} e^{-t / t_{2}}\right)$, to fit the data. The solid lines in Fig. 2(b) are fits with the fitting parameters $t_{1}=18.4 \pm 0.2 \mathrm{ps}$, $t_{2}=80 \pm 2 \mathrm{ps}$, and $A_{21}=0.19 \pm 0.01$ for the cocircular polarizations, and $t_{1}=16.8 \pm 0.3 \mathrm{ps}, t_{2}=66 \pm 2 \mathrm{ps}$, and $A_{21}=0.20 \pm 0.02$ for the cross-circular polarizations. The fit to the biexponential decay function allows a global characterization of the decay time constants for all data, however, it does not necessarily suggest that there are only two relaxation processes or attribute the time constants to a particular process.

A number of exciton decay mechanisms have been suggested as possible underlying processes in previously published studies [31-43]. For instance, some experiments [36,40] show that the fast decay dynamics $(<50 \mathrm{ps})$ is dependent on the exciton density and exciton-exciton annihilation is the dominating decay channel, while other studies [33,34,41] show no significant variation in the decay dynamics with the excitation density and that trapping by surface defect states is the responsible process. Other mechanisms such as 

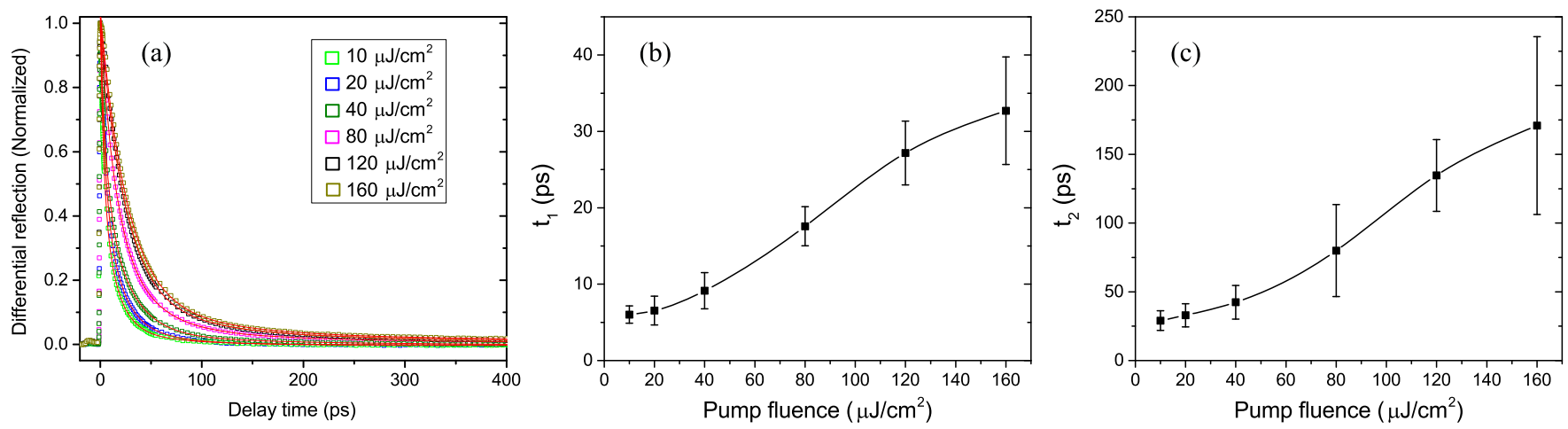

FIG. 3. (a) Normalized pump-probe spectra obtained at $10 \mathrm{~K}$ with the pump fluences ranging from 10 to $160 \mu \mathrm{J} / \mathrm{cm}^{2}$. The solid lines are fits to the biexponential decay function. (b) The extracted values of the time constant $t_{1}$ at different pump fluences. (c) The extracted values of the time constat $t_{2}$ at different pump fluences.

carrier-phonon scattering, inter/intravalley scattering, biexciton formation, trion formation, electron-hole recombination, and exciton Auger scattering have also been considered [52]. Some of the exciton decay mechanisms can also be applicable to the trion dynamics. For instance, trapping by shallow and deep defect states can lead to the biexponential decay observed in the trion dynamics. The studies on exciton dynamics [31-43] have shown a considerable discrepancy in the interpretation of the exciton dynamics and the specific time scales due to the differences in samples and measurement conditions, such as the excitation energy, excitation density, temperature, and polarizations. Particularly, the dependence on the excitation density can provide crucial evidence in determining the decay mechanism.

To further understand the trion relaxation dynamics in our sample, pump-probe spectra were obtained with the pump fluence varying from 10 to $160 \mu \mathrm{J} / \mathrm{cm}^{2}$ to investigate the excitation-density dependence. The injected carrier density is estimated to be $1.8 \times 10^{12} \mathrm{~cm}^{-2}$ for a pump fluence of $10 \mu \mathrm{J} / \mathrm{cm}^{2}$ by using an absorption measurement and assuming that one electron is excited into the conduction band for each absorbed photon. The normalized pump-probe spectra at $10 \mathrm{~K}$ with various pump fluences are shown in Fig. 3(a). The squares with different colors are experimental data and solid lines are fits to the biexponential decay function. The decay dynamics shows a strong dependence on the pump fluence, thus the initial excitation density. The extracted time constants $t_{1}$ and $t_{2}$ are plotted in Figs. 3(b) and 3(c), respectively, as a function of the pump fluence. The error bars are estimated from the fitting uncertainties and multiple measurements. Both time constants increase with the pump fluence, indicating that the trion decay is slower at higher excitation densities. This dependence is surprising since the dynamics is usually expected to be faster due to stronger many-body interactions as the density increases.

For comparison, the exciton decay dynamics was measured in a similar pump-probe experiment with the probe pulse tuned to the $A$ exciton resonance ( $1653 \mathrm{meV})$. A typical pump-probe spectrum at $10 \mathrm{~K}$ with a pump fluence of $100 \mu \mathrm{J} / \mathrm{cm}^{2}$ is shown in Fig. 4(a). The exciton decay dynamics also includes a fast and a slow decay, and can be fit to a biexponential decay function (red solid line). The extracted time constants are $t_{1}=$ $3.23 \pm 0.13 \mathrm{ps}$ and $t_{2}=235 \pm 11 \mathrm{ps}$. The measurements were repeated for different pump fluences and the corresponding time constants are shown in Fig. 4(b). Both fast and slow decay time constants essentially have no dependence on the pump fluence within the measured range. The result of the exciton dynamics is distinct from the trion dynamics, suggesting that the observed trion dynamics is not universal but unique to the trion state. The time constant $t_{2}$ is longer for excitons than
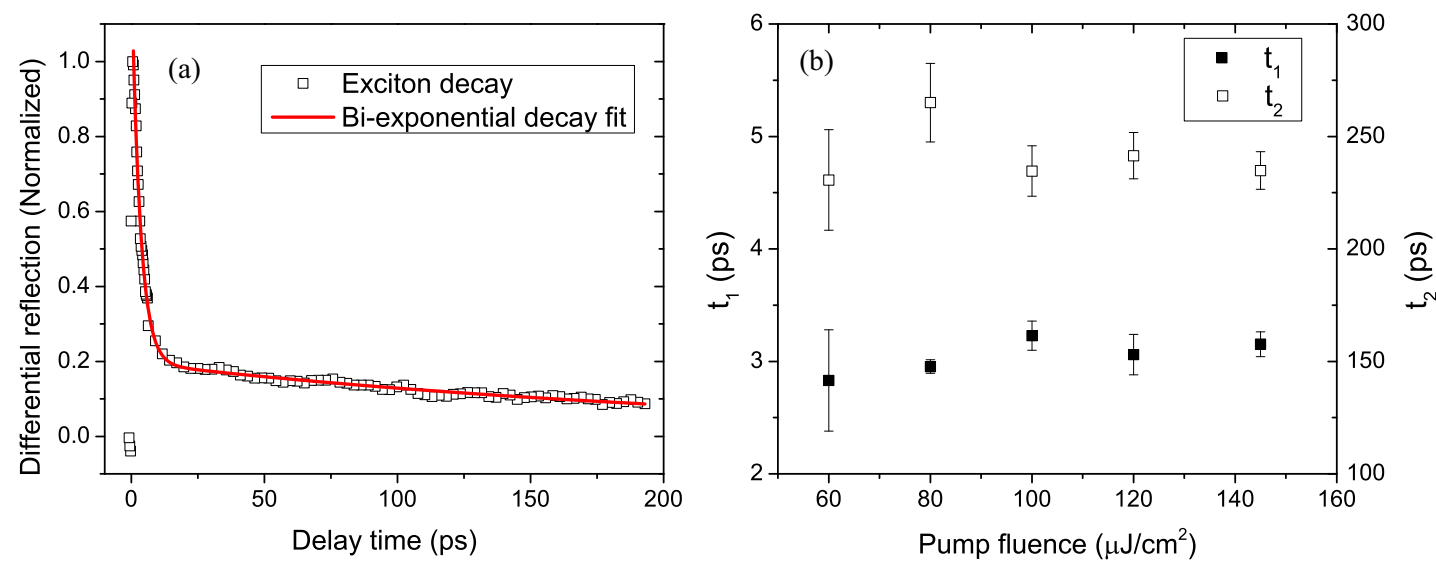

FIG. 4. (a) Normalized pump-probe spectrum of the exciton resonance at $10 \mathrm{~K}$ with a pump fluence of $100 \mu \mathrm{J} / \mathrm{cm}^{2}$. The solid line is a fit to the biexponential decay function. (b) The extracted values of the time constants $t_{1}$ and $t_{2}$ at different pump fluences. 
trions, indicating that the trion formation from the exciton is a slow process.

For exciton dynamics, the excitation-density dependence can be a result of different processes such as biexciton formation [53,54], exciton-exciton annihilation [36,40], and defect-assisted Auger scattering [41,55]. Similar processes may contribute to the trion dynamics. We rule out the biexciton formation since there is no evidence of a biexciton in monolayer $\mathrm{MoSe}_{2}$ despite the observation of biexcitons in $\mathrm{WSe}_{2}$ [53] and $\mathrm{WS}_{2}$ [54]. In a possible trion-trion annihilation process, the trion density should decay quadratically according to the rate equation $d N / d t=-k N^{2}$, with $N$ being the trion density and $k$ the annihilation rate. The decay governed by this equation should become faster as the initial density increases, opposite to the excitation-density dependence of the dynamics in our experiment. Therefore, we also rule out the trion-trion annihilation process.

The biexponential decay and the excitation-density dependence of the trion dynamics can be explained by the trapping of trions in the defect states through Auger scattering. Similar to the trapping of carriers and excitons, a trion can be captured into a defect state through either a phonon-assisted process or an Auger process. With phonon-assisted processes, the trapping rates depend strongly on the temperature. Our measurements at different temperatures up to $40 \mathrm{~K}$ (see the Supplemental Material) do not show such a temperature dependence, suggesting that phonon-assisted processes are negligible in our case. In an Auger scattering process, an electron (hole) scatters off a hole (electron) and is captured into a defect state while the hole (electron) is scattered to a higher energy state to conserve energy. The corresponding exciton or trion is trapped in the defect state. The defect trapping has been experimentally studied in $\mathrm{MoS}_{2}$ monolayers [41] and nanoclusters [56]. Two defect states have been observed to provide both fast and slow traps, which can explain the biexponential decay. In $\mathrm{MoS}_{2}$ monolayers, the decay dynamics was found [41] to be independent of the excitation density within a range of the pump fluence from 1 to $32 \mu \mathrm{J} / \mathrm{cm}^{2}$. However, the density dependence of the dynamics in our experiment can be explained by considering the limited density of the available defect states in the sample. As the exciton/trion density approaches or becomes higher than the defect density, the defect states are filled up and the defect trapping process slows down. If this is the dominating decay channel, the trion relaxation becomes slower as the initial density increases.

\section{THEORETICAL MODEL}

To test this hypothesis, we model the trion relaxation dynamics with rate equations based on the energy-level structure shown in Fig. 5(a). The exciton, trion, and ground states are considered in the excitonic picture. The excitons decay through both radiative and nonradiative channels at the rates $\Gamma_{r}^{X}$ and $\Gamma_{n r}^{X}$, respectively. The excitons can also capture an extra charge to form trions at the rate $\Gamma_{X T}$. The trions decay through the radiative recombination at the rate $\Gamma_{r}^{T}$ and the defect trapping. We assume that there are two defect states providing fast trapping at the rate $\Gamma_{f}^{T}$ and slow trapping at the rate $\Gamma_{s}^{T}$. The dynamics can be described by a set of rate (a)

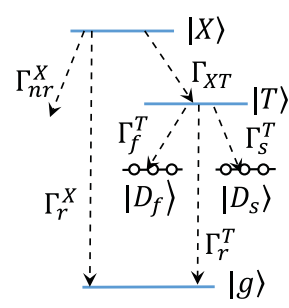

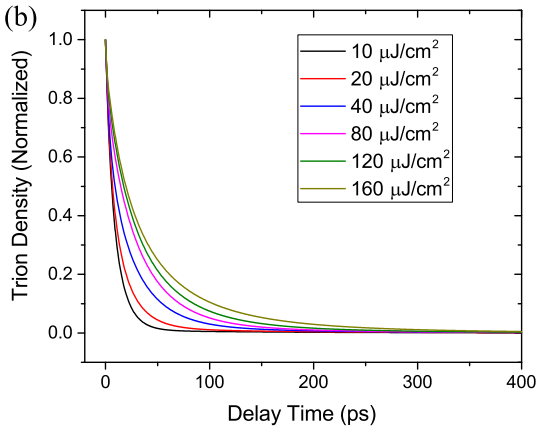

FIG. 5. (a) The energy-level diagram showing the exciton and trion decay channels. (b) The simulated trion decay dynamics for different pump fluences.

equations

$\dot{N}_{X}=-\Gamma_{r}^{X} N_{X}-\Gamma_{n r}^{X}\left(1-\frac{N_{n r}}{D_{n r}}\right) N_{X}-\Gamma_{X T}\left(1-\frac{N_{T}}{D_{T}}\right) N_{X}$,

$\dot{N}_{T}=-\Gamma_{r}^{T} N_{T}-\Gamma_{f}^{T}\left(1-\frac{N_{f}}{D_{f}}\right) N_{T}$

$$
-\Gamma_{s}^{T}\left(1-\frac{N_{s}}{D_{s}}\right) N_{T}+\Gamma_{X T}\left(1-\frac{N_{T}}{D_{T}}\right) N_{X},
$$

$\dot{N}_{f}=\Gamma_{f}^{T}\left(1-\frac{N_{f}}{D_{f}}\right) N_{T}$,

$\dot{N}_{s}=\Gamma_{s}^{T}\left(1-\frac{N_{s}}{D_{s}}\right) N_{T}$,

where $N_{X}$ is the exciton density, $N_{n r}$ is the occupied density in the nonradiative state, $D_{n r}$ is the available nonradiative state density, $N_{T}$ is the trion density, $N_{f}\left(N_{s}\right)$ is the occupied density in the fast (slow) state, $D_{f}\left(D_{S}\right)$ is the defect density for the fast (slow) trapping, and $D_{T}$ is the density of the available trion states. The photoexcited carriers form excitons and trions in a time shorter than the pulse duration. The initial exciton and trion densities are estimated from the injected carrier density and the ratio of exciton to trion density obtained from the PL spectra with the corresponding pump fluence (see the Supplemental Material). The excitons have a fast decay channel due to nonradiative processes and a slow radiative decay channel. The excitons also form trions at a time scale of hundreds of ps. The trion-to-exciton upconversion [28] has a low efficiency and is negligible in our experiment. The exciton decay rates are estimated from the exciton pumpprobe data. The rate equations are solved numerically for the trion density as a function of time. The parameters are adjusted by a nonlinear fitting routine to fit all six decay curves in Fig. 3(a) simultaneously. The simulated trion decay dynamics under different pump fluences are presented in Fig. 5(b). The corresponding fitting parameters are shown in Table I.

The simulated result is in good agreement with the experimental data. The simulation reproduces the nonexponential decay and the decay dynamics becomes slower as the pump fluence increases. We find that the density dependence of the trion dynamics is mostly affected by the defect densities $D_{f}$ 
TABLE I. The values of the parameters used in the simulation to fit the experimental data.

\begin{tabular}{lc}
\hline \hline Parameter & Value \\
\hline $1 / \Gamma_{f}^{T}$ & $10.3 \mathrm{ps}$ \\
$1 / \Gamma_{s}^{T}$ & $28.0 \mathrm{ps}$ \\
$1 / \Gamma_{X T}$ & $329 \mathrm{ps}$ \\
$1 / \Gamma_{r}^{T}$ & $500 \mathrm{ps}$ \\
$1 / \Gamma_{r}^{X}$ & $250 \mathrm{ps}$ \\
$D_{f}$ & $1.58 \times 10^{12} \mathrm{~cm}^{-2}$ \\
$D_{s}$ & $2.98 \times 10^{13} \mathrm{~cm}^{-2}$ \\
$D_{T}$ & $5 \times 10^{13} \mathrm{~cm}^{-2}$ \\
\hline \hline
\end{tabular}

and $D_{s}$. The best fitting requires $D_{f}\left(D_{s}\right)$ to be close to the lowest (highest) injected carrier density. The obtained defect densities are comparable with the theoretical and experimental values [57-60] of the point defects in 2D TMD. Although the trapping rates $\Gamma_{f}^{T}$ and $\Gamma_{s}^{T}$ stay the same for all pump fluences, the actual decay times are longer at higher densities since the defect states are filled up and the effective trapping rates $\Gamma_{f / s}^{T}\left(1-N_{f / s} / D_{f / s}\right)$ decrease with the occupied density. The fast and slow trapping by the defect states explains the nonexponential decay and the density dependence in the experimental results, but slightly overestimates the decay rate at long delay times ( $>100 \mathrm{ps}$ ) for high pump fluences $\left(\geqslant 80 \mu \mathrm{J} / \mathrm{cm}^{2}\right)$. Adding a slow process of trion formation from excitons at the rate $\Gamma_{X T}$ provides a better fit to the decay dynamics at long delay times. However, this process is not essential to reproducing the density dependence of the trion dynamics. Therefore, the trion decay dynamics is dominated by the fast and slow trapping by the defect states within the range of the pump fluences used in our experiment.

\section{CONCLUSION}

We have studied the trion dynamics in monolayer $\mathrm{MoSe}_{2}$ at a temperature of $10 \mathrm{~K}$ by using ultrafast pump-probe spectroscopy. The time scale of the trion formation from photoexcited free carriers is within $500 \mathrm{fs}$. The trion population decays nonexponentially and the decay signals can be fit with a biexponential decay function with a fast and slow time constant. Both time constants increase as the pump fluence increases, indicating slower trion decay dynamics at higher excitation densities. The nonexponential decay dynamics and its unconventional density dependence is attributed to the trapping by two defect states assisted by Auger scattering. The simulation based on the rate equations describing the trapping by defects and the exciton-to-trion formation process reproduces the measured trion decay dynamics for all pump fluences. The slower dynamics at higher densities is due to the limited defect densities that are comparable to the excitation densities. Our experiment and simulation suggest that the dominating mechanism for the trion decay dynamics is the trapping by two defect states at low temperatures within the range of the pump fluences used in our experiment.

\section{ACKNOWLEDGMENTS}

H.L. would like to thank G. Moody for useful discussions. The work at Florida International University (FIU) was supported by the Office of Research and Economic Development at FIU.
[1] K. S. Novoselov, D. Jiang, F. Schedin, T. J. Booth, V. V. Khotkevich, S. V. Morozov, and A. K. Geim, Proc. Natl. Acad. Sci. USA 102, 10451 (2005).

[2] W. Jin, P.-C. Yeh, N. Zaki, D. Zhang, J. T. Sadowski, A. AlMahboob, A. M. van der Zande, D. A. Chenet, J. I. Dadap, I. P. Herman, P. Sutter, J. Hone, and R. M. Osgood, Phys. Rev. Lett. 111, 106801 (2013).

[3] Y. Zhang, T.-R. Chang, B. Zhou, Y.-T. Cui, H. Yan, Z. Liu, F. Schmitt, J. Lee, R. Moore, Y. Chen, H. Lin, H.-T. Jeng, S.-K. Mo, Z. Hussain, A. Bansil, and Z.-X. Shen, Nat. Nanotechnol. 9, 111 (2014).

[4] K. F. Mak, C. Lee, J. Hone, J. Shan, and T. F. Heinz, Phys. Rev. Lett. 105, 136805 (2010).

[5] A. Splendiani, L. Sun, Y. Zhang, T. Li, J. Kim, C.-Y. Chim, G. Galli, and F. Wang, Nano Lett. 10, 1271 (2010).

[6] D. Xiao, G. B. Liu, W. Feng, X. Xu, and W. Yao, Phys. Rev. Lett. 108, 196802 (2012).

[7] T. Cao, G. Wang, W. Han, H. Ye, C. Zhu, J. Shi, Q. Niu, P. Tan, E. Wang, B. Liu, and J. Feng, Nat. Commun. 3, 887 (2012).

[8] K. F. Mak, K. He, J. Shan, and T. F. Heinz, Nat. Nanotechnol. 7, 494 (2012).

[9] H. Zeng, J. Dai, W. Yao, D. Xiao, and X. Cui, Nat. Nanotechnol. 7, 490 (2012).
[10] G. Sallen, L. Bouet, X. Marie, G. Wang, C. R. Zhu, W. P. Han, Y. Lu, P. H. Tan, T. Amand, B. L. Liu, and B. Urbaszek, Phys. Rev. B 86, 081301 (2012).

[11] A. M. Jones, H. Yu, N. J. Ghimire, S. Wu, G. Aivazian, J. S. Ross, B. Zhao, J. Yan, D. G. Mandrus, D. Xiao, W. Yao, and X. Xu, Nat. Nanotechnol. 8, 634 (2013).

[12] X. Wang, Y. Gong, G. Shi, W. L. Chow, K. Keyshar, G. Ye, R. Vajtai, J. Lou, Z. Liu, E. Ringe, B. K. Tay, and P. M. Ajayan, ACS Nano 8, 5125 (2014).

[13] Q. H. Wang, K. Kalantar-Zadeh, A. Kis, J. N. Coleman, and M. S. Strano, Nat. Nanotechnol. 7, 699 (2012).

[14] X. Xu, W. Yao, D. Xiao, and T. F. Heinz, Nat. Phys. 10, 343 (2014).

[15] Y. P. Shkolnikov, E. P. De Poortere, E. Tutuc, and M. Shayegan, Phys. Rev. Lett. 89, 226805 (2002).

[16] N. C. Bishop, M. Padmanabhan, K. Vakili, Y. P. Shkolnikov, E. P. De Poortere, and M. Shayegan, Phys. Rev. Lett. 98, 266404 (2007).

[17] W. Yao, D. Xiao, and Q. Niu, Phys. Rev. B 77, 235406 (2008).

[18] D. Xiao, W. Yao, and Q. Niu, Phys. Rev. Lett. 99, 236809 (2007).

[19] A. Rycerz, J. Tworzydlo, and C. W. J. Beenakker, Nat. Phys. 3, 172 (2007)

[20] O. Gunawan, B. Habib, E. P. De Poortere, and M. Shayegan, Phys. Rev. B 74, 155436 (2006). 
[21] M. A. Lampert, Phys. Rev. Lett. 1, 450 (1958).

[22] K. Kheng, R. T. Cox, M. Y. d'Aubigné, F. Bassani, K. Saminadayar, and S. Tatarenko, Phys. Rev. Lett. 71, 1752 (1993).

[23] M. T. Portella-Oberli, J. Berney, L. Kappei, F. Morier-Genoud, J. Szczytko, and B. Deveaud-Plédran, Phys. Rev. Lett. 102, 096402 (2009).

[24] A. Thilagam, Phys. Rev. B 55, 7804 (1997).

[25] K. F. Mak, K. He, C. Lee, G. H. Lee, J. Hone, T. F. Heinz, and J. Shan, Nat. Mater. 12, 207 (2013).

[26] J. S. Ross, S. Wu, H. Yu, N. J. Ghimire, A. M. Jones, G. Aivazian, J. Yan, D. G. Mandrus, D. Xiao, W. Yao, and X. Xu, Nat. Commun. 4, 1474 (2013).

[27] C. H. Lui, A. J. Frenzel, D. V. Pilon, Y.-H. Lee, X. Ling, G. M. Akselrod, J. Kong, and N. Gedik, Phys. Rev. Lett. 113, 166801 (2014).

[28] A. M. Jones, H. Yu, J. R. Schaibley, J. Yan, D. G. Mandrus, T. Taniguchi, K. Watanabe, H. Dery, W. Yao, and X. Xu, Nat. Phys. 12, 323 (2015).

[29] A. Singh, G. Moody, S. Wu, Y. Wu, N. J. Ghimire, J. Yan, D. G. Mandrus, X. Xu, and X. Li, Phys. Rev. Lett. 112, 216804 (2014).

[30] X. Xu, B. Sun, P. R. Berman, D. G. Steel, A. S. Bracker, D. Gammon, and L. J. Sham, Nat. Phys. 4, 692 (2008).

[31] T. Korn, S. Heydrich, M. Hirmer, J. Schmutzler, and C. Schuller, Appl. Phys. Lett. 99, 102109 (2011).

[32] D. Lagarde, L. Bouet, X. Marie, C. R. Zhu, B. L. Liu, T. Amand, P. H. Tan, and B. Urbaszek, Phys. Rev. Lett. 112, 047401 (2014).

[33] H. Shi, R. Yan, S. Bertolazzi, J. Brivio, B. Gao, A. Kis, D. Jena, H. G. Xing, and L. Huang, ACS Nano 7, 1072 (2013).

[34] Q. Wang, S. Ge, X. Li, J. Qiu, Y. Ji, J. Feng, and D. Sun, ACS Nano 7, 11087 (2013).

[35] Q. Cui, F. Ceballos, N. Kumar, and H. Zhao, ACS Nano 8, 2970 (2014).

[36] N. Kumar, Q. Cui, F. Ceballos, D. He, Y. Wang, and H. Zhao, Phys. Rev. B 89, 125427 (2014).

[37] C. Mai, A. Barrette, Y. Yu, Y. G. Semenov, K. W. Kim, L. Cao, and K. Gundogdu, Nano Lett. 14, 202 (2014).

[38] C. Mai, Y. G. Semenov, A. Barrette, Y. Yu, Z. Jin, L. Cao, K. W. Kim, and K. Gundogdu, Phys. Rev. B 90, 041414 (2014).

[39] E. M. Mannebach, K.-A. N. Duerloo, L. A. Pellouchoud, M.-J. Sher, S. Nah, Y.-H. Kuo, Y. Yu, A. F. Marshall, L. Cao, E. J. Reed, and A. M. Lindenberg, ACS Nano 8, 10734 (2014).

[40] D. Sun, Y. Rao, G. A. Reider, G. Chen, Y. You, L. Brézin, A. R. Harutyunyan, and T. F. Heinz, Nano Lett. 14, 5625 (2014).

[41] G. Wang, X. Marie, I. Gerber, T. Amand, D. Lagarde, L. Bouet, M. Vidal, A. Balocchi, and B. Urbaszek, Phys. Rev. Lett. 114, 097403 (2015).
[42] X.-X. Zhang, Y. You, S. Y. F. Zhao, and T. F. Heinz, Phys. Rev. Lett. 115, 257403 (2015).

[43] A. Singh, G. Moody, K. Tran, M. E. Scott, V. Overbeck, G. Berghäuser, J. Schaibley, E. J. Seifert, D. Pleskot, N. M. Gabor, J. Yan, D. G. Mandrus, M. Richter, E. Malic, X. Xu, and X. Li, Phys. Rev. B 93, 041401 (2016).

[44] L. Yang, W. Chen, K. M. McCreary, B. T. Jonker, J. Lou, and S. A. Crooker, Nano Lett. 15, 8250 (2015).

[45] L. Yang, N. A. Sinitsyn, W. Chen, J. Yuan, J. Zhang, J. Lou, and S. A. Crooker, Nat. Phys. 11, 830 (2015).

[46] G. Moody, C. Kavir Dass, K. Hao, C.-H. Chen, L.-J. Li, A Singh, K. Tran, G. Clark, X. Xu, G. Berghauser, E. Malic, A. Knorr, and X. Li, Nat. Commun. 6, 8315 (2015).

[47] J. R. Schaibley, T. Karin, H. Yu, J. S. Ross, P. Rivera, A. M. Jones, M. E. Scott, J. Yan, D. G. Mandrus, W. Yao, K.-M. Fu, and X. Xu, Phys. Rev. Lett. 114, 137402 (2015).

[48] G. Wang, L. Bouet, D. Lagarde, M. Vidal, A. Balocchi, T. Amand, X. Marie, and B. Urbaszek, Phys. Rev. B 90, 075413 (2014).

[49] See Supplemental Material at http://link.aps.org/supplemental/ 10.1103/PhysRevB.94.245413 for the analysis of the photoluminescence spectra for different pump fluences and the density dependence of the trion dynamics at different temperatures.

[50] B. Zhu, H. Zeng, J. Dai, Z. Gong, and X. Cui, Proc. Natl. Acad. Sci. USA 111, 11606 (2014).

[51] G. Wang, E. Palleau, T. Amand, S. Tongay, X. Marie, and B. Urbaszek, Appl. Phys. Lett. 106, 112101 (2015).

[52] G. Moody, J. Schaibley, and X. Xu, J. Opt. Soc. Am. B 33, C39 (2016).

[53] Y. You, X.-X. Zhang, T. C. Berkelbach, M. S. Hybertsen, D. R. Reichman, and T. F. Heinz, Nat. Phys. 11, 477 (2015).

[54] G. Plechinger, P. Nagler, J. Kraus, N. Paradiso, C. Strunk, C. Schuller, and T. Korn, Phys. Status Solidi RRL 9, 457 (2015).

[55] H. Wang, J. H. Strait, C. Zhang, W. Chan, C. Manolatou, S. Tiwari, and F. Rana, Phys. Rev. B 91, 165411 (2015).

[56] R. Doolen, R. Laitinen, F. Parsapour, and D. F. Kelley, J. Phys. Chem. B 102, 3906 (1998).

[57] H.-P. Komsa, J. Kotakoski, S. Kurasch, O. Lehtinen, U. Kaiser, and A. V. Krasheninnikov, Phys. Rev. Lett. 109, 035503 (2012).

[58] A. M. van der Zande, P. Y. Huang, D. A. Chenet, T. C. Berkelbach, Y. You, G.-H. Lee, T. F. Heinz, D. R. Reichman, D. A. Muller, and J. C. Hone, Nat. Mater. 12, 554 (2013).

[59] D. Liu, Y. Guo, L. Fang, and J. Robertson, Appl. Phys. Lett. 103, 183113 (2013).

[60] W. Zhou, X. Zou, S. Najmaei, Z. Liu, Y. Shi, J. Kong, J. Lou, P. M. Ajayan, B. I. Yakobson, and J.-C. Idrobo, Nano Lett. 13, 2615 (2013). 